\title{
Annual fasting plasma glucose variation increases risk of cancer incidence and mortality in patients with type 2 diabetes: the Taichung Diabetes Study
}

\author{
Cheng-Chieh Lin ${ }^{1,2,3}$, Chia-Ing Li, 1,3,4, Chiu-Shong Liu, 1,2,3, Wen-Yuan Lin ${ }^{1,2}$, \\ Ching-Chu Chen ${ }^{5}$, Sing-Yu Yang ${ }^{6}$, Cheng-Chun Lee ${ }^{1,7}$ and Tsai-Chung $L i^{6,8}$
}

\footnotetext{
${ }^{1}$ School of Medicine, College of Medicine, China Medical University, Taichung, Taiwan

Departments of ${ }^{2}$ Family Medicine and ${ }^{3}$ Medical Research, China Medical University Hospital, Taichung, Taiwan

${ }^{4}$ Department of Public Health, College of Public Health, China Medical University, Taichung, Taiwan

${ }^{5}$ Division of Endocrinology and Metabolism, Department of Medicine, China Medical University Hospital, Taichung, Taiwan

${ }^{6}$ College of Public Health, Graduate Institute of Biostatistics, China Medical University, Taichung, Taiwan

${ }^{7}$ Department of Neurology, China Medical University Hospital, Taichung, Taiwan

${ }^{8}$ Department of Healthcare Administration, College of Health Science, Asia University, Taichung, Taiwan

(Correspondence should be addressed to T-C Li who is now at China Medical University, 91 Hsueh-Shih Road, Taichung 40421,

Taiwan; Email: tcli@mail.cmu.edu.tw)
}

\begin{abstract}
The study aims to examine whether the annual variations in fasting plasma glucose (FPG) measurements, represented by the coefficient of variation (CV), predict cancer incidence and mortality in the subsequent years independent of traditional risk factors of type 2 diabetic patients. A computerized database of patients with type 2 diabetes of 30 years old and older $(n=4805)$ enrolled in the Diabetes Care Management Program of a medical center before 2006 was analyzed using a time-dependent Cox's proportional hazards regression model. The mortality rates for the first, second, and third tertiles of the first annual FPG-CV were 8.64, 12.71, and 30.82 per 1000 person-years respectively. After adjusting for mean FPG, HbA1c, and other risk factors, the annual FPG-CV was independently associated with cancer incidence, cancer mortality, and cancer incidence or mortality, and the corresponding hazard ratios for the third vs first tertile of the annual FPG-CV were $3.03(1.98,4.65), 5.04(2.32,10.94)$, and $2.86(1.91,4.29)$ respectively. The annual variation in FPG was a strong predictor of cancer incidence and mortality in type 2 diabetic patients; therefore, glucose variation may be important in the clinical practice of care management and cancer prevention.
\end{abstract}

Endocrine-Related Cancer (2012) 19 473-483

\section{Introduction}

Recently, cohort and case-control studies proposed a link between type 2 diabetes and cancer, especially in the colon (Larsson et al. 2005, Limburg et al. 2006, Flood et al. 2010, He et al. 2010, Hemminki et al. 2010), liver (Fujino et al. 2001, El-Serag et al. 2004), pancreatic (Gullo et al. 1994, Wang et al. 2003), breast (Mink et al. 2002, Michels et al. 2003), and prostate (Rodriguez et al. 2005, Waters et al. 2009, Li et al. 2010). Type 2 diabetes mellitus (DM) and many types of cancer share common risk factors, such as smoking, alcohol consumption, obesity, physical inactivity, and high caloric intake, particularly saturated fat intake (World Cancer Research Fund/American Institute for Cancer Research (WCRF/AICR) 1997). In addition, some possible biological mechanisms show the independent contribution of diabetes as a risk factor for cancer. These findings indicate that increased levels of insulin-like growth factor 1 (IGF1) in the serum and plasma act as a promoter of tumor cell growth. Epidemiological and experimental evidence shows the association between IGF1 and colorectal, prostate, and breast cancers (Bohlke et al. 1998, Chan et al. 1998, Cohen et al. 2000, Grimberg \& Cohen 2000). 
Moreover, recent bench studies show that the dysregulation of tuberous sclerosis 1 (TSC1)/TSC2/mTOR signaling pathway by IKB kinase (IKKB) is a common molecular switch for both cancer pathogenesis and diet- and obesity-induced insulin resistance and links obesity-derived chronic inflammation with insulin resistance and cancer pathogenesis (Greten et al. 2004, Hu et al. 2004, Lee et al. 2004, 2008).

Glucose stability is one of the classical risk factors that has been explored to explain the increasing mortality and morbidity of diabetes. Two studies by the same investigators examined the relationship of a 3 -year variation in fasting plasma glucose (FPG), measured by coefficient of variation (CV), to cancer mortality in diabetic patients $\geq 65$ years old (Muggeo et al. 1995) and 56-74 years (Muggeo et al. 2000). Although they failed to detect its ability to predict cancer mortality rates during a 5-year follow-up period, they observed the relationship of cancer mortality with FPG variation during a 10-year follow-up period. However, they did not adjust for several important comorbidities and complications or consider cancer incidence. In addition, they did not determine whether glycemic variation is also an important predictor of cancer incidence and mortality in younger patients with type 2 diabetes.

The Taichung Diabetes Study is a population-based cohort study of more than 5000 middle-aged and older Chinese patients with type 2 diabetes enrolled into the Diabetes Care Management Program (DCMP). DCMP, established by the Taiwan Bureau of National Health Insurance (BNHI) in 2002, provides financial incentives for physicians to increase follow-up visits, including annual eye examinations, annual self-care education and assessment, and annual laboratory tests, such as fasting lipid profiles, HbA1c, and FPG. DCMP provides a unique opportunity to quantify the overall effect of annual FPG variations on cancer-specific incidence and mortality. Thus, this study examines the association between annual glucose variation, as measured by the CV of all FPG measurements within each year and the cancer-specific incidence and mortality of a large number of type 2 diabetes patients of 30 years old and above who were followed for an average of 4.56 years.

\section{Materials and methods}

A retrospective cohort study was conducted on enrollees into the DCMP of China Medical University Hospital (CMUH), Taichung, Taiwan. The BNHI set up the DCMP as a nursing case management program in 2002. The enrollees were patients diagnosed with DM based on the American Diabetes Association criteria (International Classification Disease, Ninth Revision, Clinical Modification, abbreviated as ICD-9-CM; Code of 250). Upon entering the DCMP, the enrollees received a series of medical tests for blood, urine, body measurements, and their lifestyle and medical history were obtained (using a standardized computerized questionnaire administered by a case management nurse). The patients had to follow the guidelines for the managed care of diabetes proposed by the BNHI.

We identified patients with type 2 diabetes enrolled into the DCMP program in CMUH via an automated registry at the end of August 2006. The inclusion criteria were patients enrolled into the registry during the study period (August 2002 to August 2006) that were available for at least 1 year of follow-up for estimating FPG variability and 1 year of follow-up for determining the outcomes. The baseline was set as 1 year after the entry date, and the endpoint was set as August 2008, or until the patient's death. The exclusion criteria were patients with cancer at the beginning of the study and those with type 1 diabetes (ICD-9-CM; Code of $250 . x 1 / x 3$ ). A total of 4805 continuously enrolled patients with type 2 diabetes fulfilled these criteria. The current study was approved by the Ethical Review Board of CMUH.

After a $12 \mathrm{~h}$ overnight fast, blood was drawn from the antecubital vein, with minimal trauma, and was sent for analysis within $4 \mathrm{~h}$ after collection. Biochemical markers, such as FPG, high-density lipoprotein cholesterol (HDL-C), and triglyceride were analyzed by a biochemical autoanalyzer (Beckman Coulter Synchron system, Lx-20, Fullerton, CA, USA) at the Clinical Laboratory Department of CMUH. For both interassay and intra-assay CV, FPG were both $4 \%$. Plasma cholesterol and triglyceride levels were determined via an enzymatic colorimetric method. The HDL-C level was measured using a direct HDL-C method and the low-density lipoprotein cholesterol (LDL-C) level was measured by a direct LDL-C method. The interassay and intra-assay $\mathrm{CV}$ for triglyceride were 6.8 and 5\% respectively; the interassay and intra-assay CV for HDL-C were 4.5 and $4.5 \%$ respectively; and the interassay and intra-assay CV for LDL-C were 4.5 and $3 \%$ respectively.

The primary outcomes were measured in terms of cancer incidence and mortality. Taiwan's National Death Index, a file tallying the deaths of all citizens based on death certificates, was used to identify possible decedents with cancers during the follow-up period. After the deaths were identified, they were confirmed through the cancer registry of CMUH. By cross-referencing the unique identification number 
Table 1 The comparisons of sociodemographic factors, lifestyle behaviors, diabetes-related variables, drug-related variables, diabetes-related diseases, and blood biochemical indexes according to cancer-specific incidence or mortality with type 2 diabetes enrolled in the Diabetes Care Management Program at the CMUH, Taichung, Taiwan $(n=4805)$

\begin{tabular}{|c|c|c|c|}
\hline \multirow[b]{2}{*}{ Variables } & \multicolumn{2}{|c|}{ Cancer incidence or mortality cases $\boldsymbol{n}(\%)$} & \multirow[b]{2}{*}{$P$ value } \\
\hline & No $(n=4618)$ & Yes $(n=187)$ & \\
\hline \multicolumn{4}{|l|}{ Sociodemographic factors } \\
\hline Gender & & & 0.07 \\
\hline Female & $2278(49.33)$ & $79(42.25)$ & \\
\hline Male & $2340(50.67)$ & $108(57.75)$ & \\
\hline Age (years) $^{\mathrm{a}}$ & $58.47(11.47)$ & $65.10(10.34)$ & $<0.001$ \\
\hline \multicolumn{4}{|l|}{ Lifestyle behaviors } \\
\hline Smoking & & & 0.99 \\
\hline No & $3764(81.51)$ & $153(81.82)$ & \\
\hline Yes & $854(18.49)$ & $34(18.18)$ & \\
\hline Alcohol drinking & & & 0.19 \\
\hline No & $4119(89.19)$ & $173(92.51)$ & \\
\hline Yes & $499(10.81)$ & $14(7.49)$ & \\
\hline \multicolumn{4}{|l|}{ Diabetes-related variables } \\
\hline Duration of diabetes medication (years) ${ }^{a}$ & $6.97(7.04)$ & $8.38(7.66)$ & 0.01 \\
\hline Type of DM treatment & & & 0.64 \\
\hline Oral hypoglycemic drug & $3787(82.01)$ & $157(83.96)$ & \\
\hline Inject insulin & $79(1.71)$ & $1(0.53)$ & \\
\hline Both & $454(9.83)$ & $18(9.63)$ & \\
\hline Both not & $298(6.45)$ & $11(5.88)$ & \\
\hline \multicolumn{4}{|l|}{ Drug-related variables } \\
\hline Hypertension drug treatment & & & 1.00 \\
\hline No & $2573(55.72)$ & 104 (55.61) & \\
\hline Yes & $2045(44.28)$ & 83 (44.39) & \\
\hline \multicolumn{4}{|l|}{ Diabetes-related diseases } \\
\hline Obesity & & & 0.92 \\
\hline No & $3141(68.02)$ & $126(67.38)$ & \\
\hline Yes & $1477(31.98)$ & $61(32.62)$ & \\
\hline Hypertension & & & 1.00 \\
\hline No & $2597(56.24)$ & $105(56.15)$ & \\
\hline Yes & $2021(42.98)$ & $82(43.85)$ & \\
\hline Hyperlipidemia & & & 0.90 \\
\hline No & $3145(68.10)$ & $126(67.38)$ & \\
\hline Yes & $1473(31.90)$ & 61 (32.62) & \\
\hline DKA & & & 0.73 \\
\hline No & 4559 (98.72) & $186(99.47)$ & \\
\hline Yes & $59(1.28)$ & $1(0.53)$ & \\
\hline HHNK & & & 1.00 \\
\hline No & $4515(97.77)$ & $183(97.86)$ & \\
\hline Yes & $103(2.23)$ & $4(2.14)$ & \\
\hline Severe hypoglycemia & & & 0.80 \\
\hline No & $4508(97.62)$ & $184(98.40)$ & \\
\hline Yes & $110(2.38)$ & $3(1.60)$ & \\
\hline Stroke & & & 0.04 \\
\hline No & $4275(92.57)$ & $165(88.24)$ & \\
\hline Yes & $343(7.43)$ & $22(11.76)$ & \\
\hline Coronary artery disease & & & 1.00 \\
\hline No & $4265(92.36)$ & $173(92.51)$ & \\
\hline Yes & $353(7.64)$ & $14(7.49)$ & \\
\hline Myocardial infarction & & & 0.19 \\
\hline No & 4259 (92.23) & 167 (89.30) & \\
\hline Yes & $359(7.77)$ & $20(10.70)$ & \\
\hline Peripheral neuropathy & & & 1.00 \\
\hline No & $3914(84.76)$ & $158(84.49)$ & \\
\hline Yes & $704(15.24)$ & $29(15.51)$ & \\
\hline
\end{tabular}




\begin{tabular}{|c|c|c|c|}
\hline \multirow[b]{2}{*}{ Variables } & \multicolumn{2}{|c|}{ Cancer incidence or mortality cases $\boldsymbol{n}(\%)$} & \multirow[b]{2}{*}{$P$ value } \\
\hline & No $(n=4618)$ & Yes $(n=187)$ & \\
\hline Intermittent claudication & & & 0.52 \\
\hline No & $4552(98.57)$ & $186(99.46)$ & \\
\hline Yes & $66(1.43)$ & $1(0.53)$ & \\
\hline Neuropathy & & & 0.36 \\
\hline No & 4248 (91.99) & $168(89.84)$ & \\
\hline Yes & $370(8.01)$ & $19(10.16)$ & \\
\hline Nephropathy & & & 0.46 \\
\hline No & $4132(89.48)$ & $171(91.44)$ & \\
\hline Yes & 486 (10.52) & $16(8.56)$ & \\
\hline \multicolumn{4}{|l|}{ Blood biochemical indexes } \\
\hline Triglyceride $(\mathrm{mg} / \mathrm{dl})^{\mathrm{a}}$ & $166.90(217.20)$ & $148.70(114.30)$ & 0.04 \\
\hline High-density lipoprotein $(\mathrm{mg} / \mathrm{dl})^{\mathrm{a}}$ & $41.06(11.31)$ & $39.71(9.64)$ & 0.07 \\
\hline $\mathrm{HbA1c}(\%)^{\mathrm{a}}$ & $8.26(1.90)$ & $8.20(1.76)$ & 0.64 \\
\hline Fasting plasma glucose $(\mathrm{mg} / \mathrm{dl})^{\mathrm{a}}$ & $163.90(59.02)$ & $159.20(53.83)$ & 0.28 \\
\hline
\end{tabular}

DKA, diabetic ketoacidosis; HHNK, hyperglycemic hyperosmolar non-ketoacidosis. Differences in continuous variables were tested by Student's $t$-test. Differences in categorical variables were tested by the $\chi^{2}$ test.

Mean \pm s.D.

with this computerized file, 70 cancer deaths were identified from this cohort by 2008 . The deaths were classified according to the International Classification of Diseases, 9th Revised Edition. Mortality from malignant neoplasms was defined as codes 140-208. Cancer incidence cases were identified via crossreferencing with cancer registry and via follow-up visits. A total of 172 new DM type 2 cases were identified, 55 of which died of cancer during the follow-up period. Thus, 187 new cases or deaths were included in the analysis.

\section{Statistical analysis}

The annual CV of all FPG measurements within each year was computed for each patient using the dataset from electronic laboratory records. The FPG-CV was calculated only when the three or more FPG measurements were recorded within each year. The patients were grouped into tertiles based on the first year of FPG-CV.

Kaplan-Meier cumulative incidence plots were generated to show the time-to-event for all endpoints. We used the time-dependent Cox proportional hazard models to evaluate the association of FPG-CV categories with the primary outcome measures by considering time variations in FPG-CV. We calculated the hazard ratios (HR) and their $95 \%$ confidence intervals after adjusting for age and multiple variables. Three multivariate models were conducted. The first multivariate model was adjusted for age (continuous), obesity, hypertension, antihypertensive treatment (yes, no), smoking (never, past, current), exercise (yes, no), alcohol consumption (yes, no), hyperlipidemia, and type of treatment (oral hypoglycemic drug, insulin injection, both, or diet or exercise). The second model included a mean FPG, estimated glomerular filtration rate (eGFR), and HbA1c. The third model included complications at the baseline (diabetic ketoacidosis (DKA), hyperglycemic hyperosmolar non-ketoacidosis (HHNK), severe hypoglycemia, stroke, myocardial infarction (MI), peripheral neuropathy, intermittent claudication, neuropathy, and nephropathy).

To assess whether the FPG-CV estimates on cancer incidence or mortality were sensitive to diabetic complication, we excluded patients with stroke, cardiovascular disease (CVD), diabetic ketoacidosis (DKA), and hyperglycemic, hyperosmolar, nonketoacidosis (HHNK) and performed a multivariate analysis. We tested the proportionality assumption by including an interaction term for the FPG-CV categories with person-time in the Cox models and found no statistically significant violation. We calculated $P$ for the trend of the FPG-CV categories across tertiles. All analyses were performed using SAS version 9.2 (SAS, Cary, NC, USA). All $P$ values were two tailed, and a $P$ value $<0.05$ was considered statistically significant.

\section{Results}

Over an average of 4.68 years of follow-up, 187 patients with diabetes died of cancer or developed cancers, with a crude rate of $8.32 / 1000$ person-years 
Table 2 The comparisons of baseline sociodemographic factors, lifestyle behaviors, diabetes-related variables, drug-related variables, diabetes-related diseases, and blood biochemical indexes according to tertiles of coefficient of variation (CV) of baseline fasting plasma glucose with type 2 diabetes enrolled in the Diabetes Care Management Program at the CMUH, Taichung, Taiwan $(n=4805)$

\begin{tabular}{|c|c|c|c|c|}
\hline \multirow[b]{2}{*}{ Variables } & \multicolumn{3}{|c|}{ Baseline FPG-CV, $\boldsymbol{n}(\%)$} & \multirow[b]{2}{*}{$P$ value } \\
\hline & $\leq 14.41(n=1580)$ & $14.41-25.10(n=1594)$ & $>25.10(n=1631)$ & \\
\hline \multicolumn{5}{|l|}{ Sociodemographic factors } \\
\hline Gender & & & & 0.001 \\
\hline Female & $733(46.39)$ & 839 (52.63) & $785(48.13)$ & \\
\hline Male & $847(53.61)$ & $755(47.37)$ & $846(51.87)$ & \\
\hline Age (years) ${ }^{\mathrm{a}}$ & $58.31(11.11)$ & $58.45(11.11)$ & $59.42(12.18)$ & 0.01 \\
\hline \multicolumn{5}{|l|}{ Lifestyle behaviors } \\
\hline Smoking & & & & $<0.001$ \\
\hline No & $1332(84.30)$ & $1311(82.25)$ & $1274(78.11)$ & \\
\hline Yes & $248(15.70)$ & $283(17.75)$ & 357 (21.89) & \\
\hline Alcohol drinking & & & & 0.18 \\
\hline No & $1425(90.19)$ & $1406(88.21)$ & $1461(89.58)$ & \\
\hline Yes & $155(9.81)$ & 188 (11.79) & $170(10.42)$ & \\
\hline \multicolumn{5}{|l|}{ Diabetes-related variables } \\
\hline Diabetes medical history (years) ${ }^{a}$ & $5.05(5.94)$ & $7.03(6.66)$ & $8.94(7.89)$ & $<0.001$ \\
\hline Type of DM treatment & & & & $<0.001$ \\
\hline Oral hypoglycemic drug & $1361(86.14)$ & $1403(88.02)$ & $1180(72.35)$ & \\
\hline Inject insulin & $6(0.38)$ & $14(0.88)$ & $60(3.68)$ & \\
\hline Both & $42(2.66)$ & $106(6.65)$ & $324(19.87)$ & \\
\hline Diet or exercise & $171(10.82)$ & $71(4.45)$ & $67(4.11)$ & \\
\hline \multicolumn{5}{|l|}{ Drug-related variables } \\
\hline Hypertension drug treatment & & & & 0.02 \\
\hline No & $891(56.39)$ & $920(57.72)$ & $866(53.10)$ & \\
\hline Yes & $689(43.61)$ & $674(42.28)$ & $765(46.90)$ & \\
\hline \multicolumn{5}{|l|}{ Diabetes-related diseases } \\
\hline Obesity & & & & 0.001 \\
\hline No & $1019(64.49)$ & $1103(69.20)$ & $1145(70.20)$ & \\
\hline Yes & $561(35.51)$ & 491 (30.80) & $486(29.80)$ & \\
\hline Hypertension & & & & 0.01 \\
\hline No & $917(58.04)$ & $916(57.47)$ & 869 (53.28) & \\
\hline Yes & $663(41.96)$ & $678(42.53)$ & $762(46.72)$ & \\
\hline Hyperlipidemia & & & & 0.74 \\
\hline No & $1078(68.23)$ & $1094(68.63)$ & $1099(67.38)$ & \\
\hline Yes & $502(31.77)$ & 500 (31.37) & 532 (32.62) & \\
\hline DKA & & & & $<0.001$ \\
\hline No & $1568(99.24)$ & $1582(99.25)$ & 1595 (97.79) & \\
\hline Yes & $12(0.76)$ & $12(0.75)$ & $36(2.21)$ & \\
\hline HHNK & & & & $<0.001$ \\
\hline No & $1554(98.35)$ & $1569(98.43)$ & $1575(96.57)$ & \\
\hline Yes & $26(1.65)$ & $25(1.57)$ & $56(3.43)$ & \\
\hline Severe hypoglycemia & & & & $<0.001$ \\
\hline No & $1560(98.73)$ & $1558(97.74)$ & $1574(96.51)$ & \\
\hline Yes & $20(1.27)$ & $36(2.26)$ & $57(3.49)$ & \\
\hline Stroke & & & & 0.005 \\
\hline No & $1481(93.73)$ & $1497(92.79)$ & $1480(90.74)$ & \\
\hline Yes & $99(6.27)$ & $115(7.21)$ & $151(9.26)$ & \\
\hline Coronary artery disease & & & & 0.17 \\
\hline No & $1473(93.23)$ & $1473(92.41)$ & $1492(91.48)$ & \\
\hline Yes & 107 (6.77) & $121(7.59)$ & 139 (8.52) & \\
\hline Myocardial infarction & & & & 0.009 \\
\hline No & $1480(93.67)$ & 1466 (91.97) & $1480(90.74)$ & \\
\hline Yes & $100(6.33)$ & $128(8.03)$ & $151(9.26)$ & \\
\hline Peripheral neuropathy & & & & $<0.001$ \\
\hline No & $1434(90.76)$ & $1363(85.51)$ & 1275 (78.17) & \\
\hline Yes & $146(9.24)$ & $231(14.49)$ & $356(21.83)$ & \\
\hline
\end{tabular}




\begin{tabular}{|c|c|c|c|c|}
\hline \multirow[b]{2}{*}{ Variables } & \multicolumn{3}{|c|}{ Baseline FPG-CV, $\boldsymbol{n}(\%)$} & \multirow[b]{2}{*}{$P$ value } \\
\hline & $\leq 14.41(n=1580)$ & $14.41-25.10(n=1594)$ & $>25.10(n=1631)$ & \\
\hline Intermittent claudication & & & & $<0.001$ \\
\hline No & 1568 (99.24) & 1577 (98.93) & $1593(97.67)$ & \\
\hline Yes & $12(0.76)$ & $17(1.07)$ & $38(2.33)$ & \\
\hline Neuropathy & & & & $<0.001$ \\
\hline No & $1503(95.13)$ & 1479 (92.79) & 1434 (87.92) & \\
\hline Yes & $77(4.87)$ & $115(7.21)$ & 197 (12.08) & \\
\hline Nephropathy & & & & $<0.001$ \\
\hline No & $1492(94.43)$ & $1445(90.65)$ & $1366(83.75)$ & \\
\hline Yes & $88(5.57)$ & $149(9.35)$ & 265 (16.25) & \\
\hline \multicolumn{5}{|l|}{ Blood biochemical indexes } \\
\hline Triglyceride $(\mathrm{mg} / \mathrm{dl})^{\mathrm{a}}$ & $148.76(128.86)$ & $168.50(238.93)$ & $180.85(250.46)$ & $<0.001$ \\
\hline High-density lipoprotein (mg/dl) ${ }^{a}$ & $40.98(10.34)$ & $41.04(11.04)$ & $41.01(12.28)$ & 0.99 \\
\hline Mean $\mathrm{HbA} 1 \mathrm{c}$ of the first year $(\%)^{\mathrm{a}}$ & $7.19(1.10)$ & $7.87(1.31)$ & $8.60(1.52)$ & $<0.001$ \\
\hline $\begin{array}{l}\text { Mean fasting plasma glucose of the } \\
\text { first year }(\mathrm{mg} / \mathrm{dl})^{\mathrm{a}}\end{array}$ & $145.43(32.87)$ & $158.24(36.92)$ & $176.76(54.85)$ & $<0.001$ \\
\hline
\end{tabular}

Differences in continuous variables were tested by Student's $t$-test. Differences in categorical variables were tested by $\chi^{2}$ test. ${ }^{\mathrm{a}}$ Mean (S.D.).

(9.50 men and 7.10 women). Liver cancer had the highest incidence and mortality $(n=18$ for mortality and $n=34$ for incidence) followed by lung cancer ( $n=16$ for mortality and $n=21$ for incidence) and colon cancer $(n=10$ for mortality and $n=18$ for incidence). Altogether, these cases accounted for $42.25 \%$ of all cancer incidence and deaths. Table 1 summarizes the comparisons of baseline sociodemographic factors, lifestyle behaviors, diabetes-related variables, drug-related variables, comorbidities, complications, and blood biochemical indices in terms of cancer incidence and mortality of patients with diabetes who were enrolled in the DCMP of a medical center. Compared with patients who died of cancer or developed cancer during the follow-up period, the patients who did not have cancer had a younger mean age, shorter duration of diabetes medication, lower prevalence of strokes, and a higher mean triglyceride levels. Lower FPG-CV was associated with lower mean age, duration of diabetes medication, triglyceride levels, mean $\mathrm{HbA} 1 \mathrm{c}$, and FPG on the first year and lower prevalence of female gender, smoking, treatment with both oral hypoglycemic drugs and insulin injection, antihypertensive drug treatment, hypertension, DKA, HHNK, severe hypoglycemia, stroke, MI, peripheral neuropathy, intermittent claudication, neuropathy, and nephropathy (Table 2).

Figure 1 presents the Kaplan-Meier cumulative incidence of the time-to-event for cancer incidence, cancer mortality, and cancer incidence or mortality within the subgroups defined by the baseline FPG-CV.
The median follow-up time ranged from 5.22 years, for patients with FPG-CV $<14.41 \%$, to 5.13 years, for patients with FPG-CV $\geq 25.10 \%$. Baseline FPG-CV was a powerful predictor of cancer incidence, cancer mortality, and cancer incidence or mortality (Fig. 1). We found that patients with FPG-CV $\geq 25.10 \%$ have increased cancer incidence (log-rank $P<0.001$, Fig. 1a), cancer mortality (log-rank $P<0.001$, Fig. 1b), and cancer incidence or mortality (log-rank $P<0.001$, Fig. 1c). Figure 2 shows the means and standard errors of FPG-CV from years 1 to 6 based on cancer incidence and mortality. The individuals who died of cancer or developed cancer had higher FPG-CV than those without cancer incidence or mortality from years 0 to 3 and 5 (all $P<0.01$ ).

Table 3 shows the HRs for cancer incidence, cancer mortality, and cancer incidence or mortality according to the tertiles of the time-dependent annual FPG-CV. Compared with patients with annual FPG-CV $\leq 14.41 \%$, the age-adjusted HRs for cancer incidence among patients with annual FPG-CV $>25.10 \%$ were 2.79 (95\% confidence interval 1.86-4.17). For cancer mortality, the HRs were 5.32 (2.52-11.21), whereas the HRs for cancer incidence or mortality were 2.61 (1.78-3.82).

We found significant linear trends across annual FPG-CV categories for cancer incidence, cancer mortality, and cancer incidence or mortality. When the mean FPG, eGFR, and HbAlc were considered, the effect of FPG-CV remained the same and statistically significant. After adjusting further for complications, the multivariate-adjusted HR was $3.03(1.98,4.65)$ for 

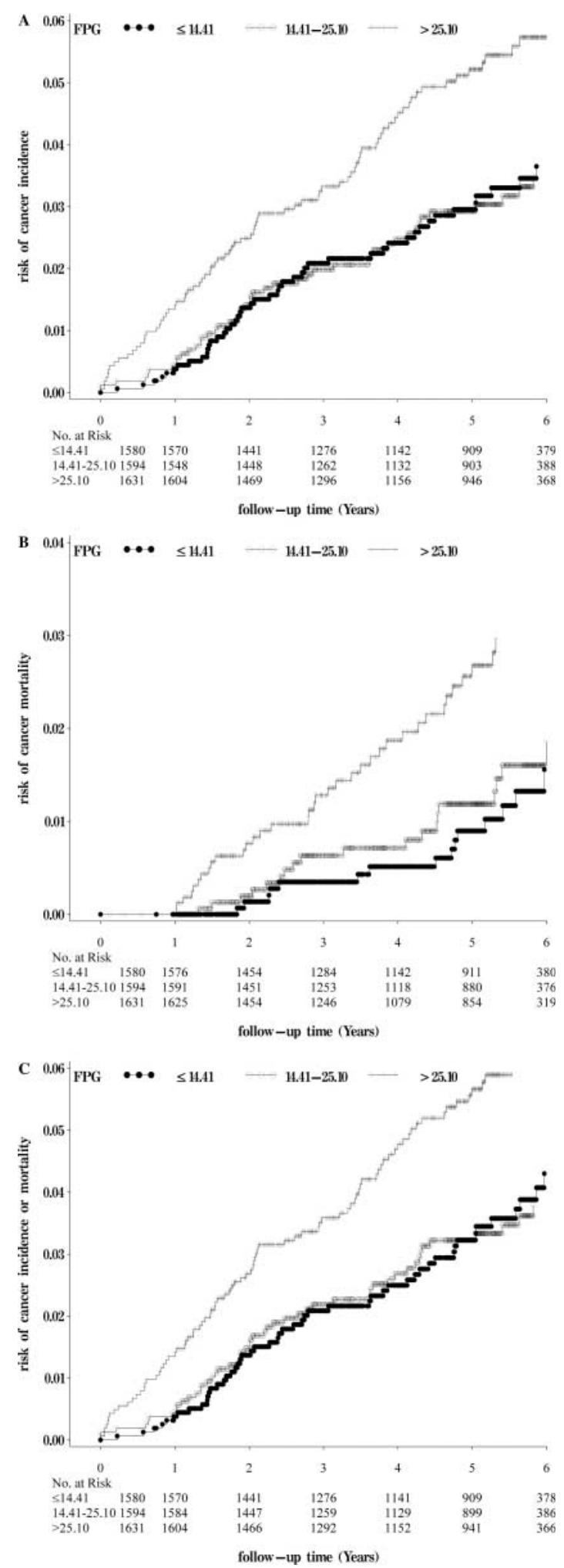

Figure 1 Risk from cancer incidence (a), cancer mortality (b), and cancer incidence or mortality (c) for baseline FPG-CV. cancer incidence, $5.04(2.32,10.94)$ for cancer mortality, and $2.86(1.91,4.29)$ for cancer incidence or mortality among patients in the third tertile compared with those in the first tertile.

Sensitivity analyses were conducted to investigate the potential for bias because of comorbidities by excluding patients who developed stroke, CVD, DKA, HHNK, and nephropathy $(n=3612)$. Similar significant associations were found for the HRs for cancer incidence $(2.91,1.82-4.65)$, cancer mortality (5.52, 2.24-13.56), and cancer incidence or mortality (2.76, 1.76-4.32) among patients in the third tertile compared with those in the first tertile.

\section{Discussion}

This study shows that the annual variation in FPG measurements predicts cancer incidence and cancer mortality among type 2 diabetes patients of 30-93 years old. Compared to patients with FPG-CV $\leq 14.41 \%$, those with FPG-CV from 14.41 to $25.10 \%$ and $>25.10 \%$ have a 1.63 - and 3 -fold higher cancer incidence and mortality respectively. These findings are relevant to the clinical management of patients with type 2 diabetes. Managed care facilitates the routine FPG measurements; hence, these routine glycemic assessments of patients with diabetes should be used for improving treatment strategies.

Consistent with the study by Muggeo et al. (2000), risk of cancer-specific mortality increased with FPG variation in our study. Instead of the time-dependent annual FPG variation, they measured the 3-year FPG variation among older patients with type 2 diabetes and observed the relationship of cancer mortality with FPG variation during 10 years of follow-up. In our study, predicting cancer mortality rates remained significantly stable when the means of FPG, eGFR, and $\mathrm{HbA} 1 \mathrm{c}$ were considered. To rule out the possibility that comorbidities and complications account for the relationships observed in the current study, we further adjusted for comorbidities and complications. The associations remained significant.

We observed a significant association between the annual FPG variation during follow-up and cancer mortality, which was consistent with the previous studies (Muggeo et al. 2000). These observed associations have three possible explanations. First, malignancies result in glucose variability through impaired feeding. To rule out this possibility, we compared the mean FPG-CV of the last annual measurement with that of the remaining ones among patients who developed cancer or died of cancer during the follow-up, which were 23.82 and $22.69 \%$ 

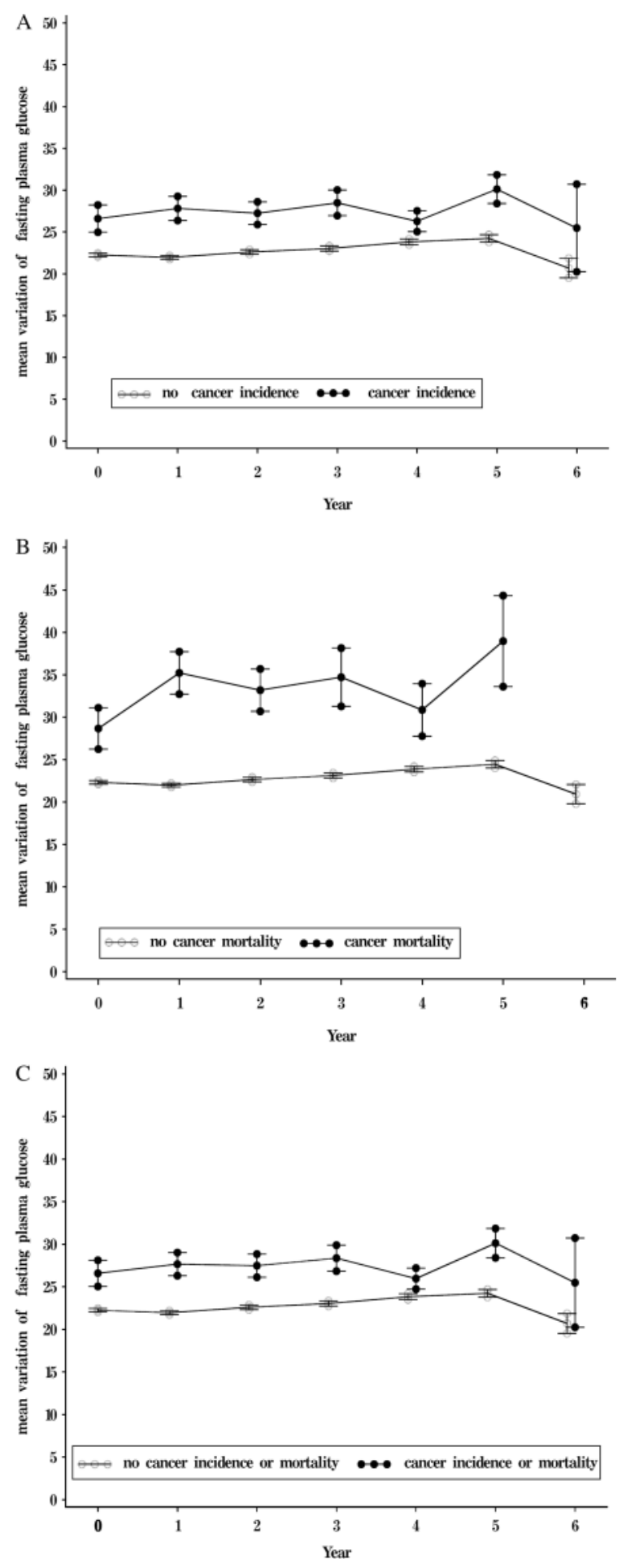

Figure 2 Mean variation of fasting plasma glucose according to cancer incidence (a), cancer mortality (b), and cancer incidence or mortality (c).

respectively. Considering the FPG-CV in the last and the remaining annual measurements of new cancer cases or those who died of cancer during the follow-up were consistently high, we could rule out that glucose variation is a predictor of cancer incidence and mortality. Secondly, although cancer treatment may have a detrimental effect on glucose control, it does not account for the relationship between diabetes and cancer because we excluded patients with cancer at the start of the study period. Thirdly, insulin resistance and the progression of cancer may share the same mechanism; therefore, glucose variability could account for this relationship better. A recent bench study showed that the dysregulation of the TSC1/ TSC2/mTOR signaling pathway by IKKB is a common molecular switch for both cancer pathogenesis and diet- and obesity-induced insulin resistance and links obesity-derived chronic inflammation with insulin resistance and cancer pathogenesis (Greten et al. 2004, Hu et al. 2004, Lee et al. 2004, 2008). Under this mechanism, the relationship between FPG variation and cancer mortality becomes more plausible over time.

Metabolic syndrome is a cluster of endocrinemetabolic disturbances characterized by insulin resistance, impaired glucose regulation, hypertension, raised triglycerides, and low HDL-C (Alberti et al. 2006, Cust et al. 2007). Insulin resistance caused by obesity or an inherited genetic defect has been hypothesized as the mechanism underlying metabolic syndrome. Insulin resistance is a pathological condition with reduced glycogen synthesis and storage and high levels of circulating glucose. For its role in cancer risk, insulin may act directly as a mitogenic and anti-apoptotic growth factor in tissues (Nagamani \& Stuart 1998, Cust et al. 2007). Insulin also increases IGFI bioactivity (Kaaks \& Lukanova 2001, Kaaks et al. 2002, Cust et al. 2007). The shared metabolic factors that underlie both DM2 and cancer, including visceral adiposity, inflammation, hyperglycemia, and hyperinsulinemia, lead to increased insulin receptor substrate (IRS). IRS stimulates Ras phosphorylation, which signals proteins and potentially increases tumor cell growth and proliferation. Another possible mechanism is that IRS-associated PI3K signaling is compromised by insulin-resistant states, such as in type 2 diabetes. This phenomenon results in the disruption of downstream GLUT4 translocation, which drives PI3K signaling towards AKT/mTOR. AKT and mTOR affect both the metabolic and the mitogenic pathways.

In addition, the components of metabolic syndrome have each been linked to the development of cancer. Hypertension may increase cancer risk by blocking and 
Table 3 The hazard ratios (HRs) of cancer incidence or mortality according to tertiles of time-dependent annual FPG-CV in patients with type 2 diabetes enrolled in Diabetes Care Management Program of a medical center $(n=4805)$

\begin{tabular}{|c|c|c|c|c|c|c|}
\hline Variables & $\begin{array}{c}\text { Cancer incidence } \\
(n=172) \\
\text { HR }(95 \% \mathrm{Cl})\end{array}$ & $\begin{array}{l}P \text { for } \\
\text { trend }\end{array}$ & $\begin{array}{c}\text { Cancer mortality } \\
(n=70) \\
\text { HR }(95 \% \mathrm{Cl})\end{array}$ & $\begin{array}{l}P \text { for } \\
\text { trend }\end{array}$ & $\begin{array}{c}\text { Cancer incidence or } \\
\text { mortality }(n=187) \\
\text { HR }(95 \% \mathrm{Cl})\end{array}$ & $\begin{array}{l}P \text { for } \\
\text { trend }\end{array}$ \\
\hline \multicolumn{7}{|l|}{ Age adjusted } \\
\hline FPG-CV (\%) & & $<0.001$ & & $<0.001$ & & $<0.001$ \\
\hline$\leq 14.41$ & 1.00 & & 1.00 & & 1.00 & \\
\hline $14.41-25.10$ & $1.60(0.99,2.59)$ & & $1.45(0.57,3.68)$ & & $1.58(1.00,2.48)^{*}$ & \\
\hline$>25.10$ & $2.79(1.86,4.17)^{\dagger}$ & & $5.32(2.52,11.21)^{\dagger}$ & & $2.61(1.78,3.82)^{\dagger}$ & \\
\hline \multicolumn{7}{|c|}{ Multivariate adjusted $^{\mathrm{a}}$} \\
\hline FPG-CV (\%) & & $<0.001$ & & $<0.001$ & & $<0.001$ \\
\hline$\leq 14.41$ & 1.00 & & 1.00 & & 1.00 & \\
\hline $14.41-25.10$ & $1.66(1.03,2.69)^{\star}$ & & $1.50(0.59,3.82)$ & & $1.64(1.04,2.59)^{*}$ & \\
\hline$>25.10$ & $2.95(1.96,4.45)^{\dagger}$ & & $5.44(2.54,11.62)^{\dagger}$ & & $2.78(1.88,4.10)^{\dagger}$ & \\
\hline \multicolumn{7}{|c|}{ Multivariate adjusted $^{\mathrm{b}}$} \\
\hline FPG-CV (\%) & & $<0.001$ & & $<0.001$ & & $<0.001$ \\
\hline$\leq 14.41$ & 1.00 & & 1.00 & & 1.00 & \\
\hline $14.41-25.10$ & $1.67(1.02,2.74)^{\star}$ & & $1.46(0.57,3.72)$ & & $1.64(1.03,2.62)^{*}$ & \\
\hline$>25.10$ & $3.00(1.96,4.60)^{\dagger}$ & & $5.09(2.35,11.03)^{\dagger}$ & & $2.83(1.89,4.24)^{\dagger}$ & \\
\hline \multicolumn{7}{|c|}{ Multivariate adjusted $^{\mathrm{C}}$} \\
\hline FPG-CV (\%) & & $<0.001$ & & $<0.001$ & & $<0.001$ \\
\hline$\leq 14.41$ & 1.00 & & 1.00 & & 1.00 & \\
\hline$\overline{14} .41-25.10$ & $1.66(1.01,2.71)^{*}$ & & $1.45(0.57,3.69)$ & & $1.63(1.03,2.60)^{*}$ & \\
\hline$>25.10$ & $3.03(1.98,4.65)^{\dagger}$ & & $5.04(2.32,10.94)^{\dagger}$ & & $2.86(1.91,4.29)^{\dagger}$ & \\
\hline
\end{tabular}

FPG-CV, coefficient of variation of fasting plasma glucose. ${ }^{\star} P<0.05 ;{ }^{\dagger} P<0.001$.

${ }^{a}$ Multivariate-adjusted age, smoking, alcohol drinking, hypertension, hypertension drug treatment, obesity, hyperlipidemia, and type of diabetes treatment.

${ }^{\mathrm{b}}$ Multivariate-adjusted age, smoking, alcohol drinking, hypertension, hypertension drug treatment, obesity, hyperlipidemia, type of diabetes treatment, mean annual fasting plasma glucose, estimated glomerular filtration rate, and $\mathrm{HbA} 1 \mathrm{c}$.

${ }^{c}$ Multivariate-adjusted age, smoking, alcohol drinking, hypertension, hypertension drug treatment, obesity, hyperlipidemia, type of diabetes treatment, mean annual fasting plasma glucose, estimated glomerular filtration rate, HbA1c, DKA, HHNK, severe hypoglycemia, stroke, myocardial infarction, peripheral neuropathy, intermittent claudication, neuropathy, and nephropathy.

subsequently modifying apoptosis, thereby affecting cell turnover (Mason 1999, Soler et al. 1999, Russo et al. 2008). Hyperglycemia may not only promote tumorigenesis indirectly by stimulating insulin production but may also have a direct effect because cancer cells depend on glycolysis for energy (Levine et al. 1990, Yun et al. 2009). The possible mechanism for the relationship between obesity and carcinogenesis, and the progression of multiple cancer sites, is through the signaling pathways that regulate key functions, including cancer cell proliferation, apoptosis, metastasis, and angiogenesis (Overvad 2002). Our study demonstrates that glucose variation, characteristics of diabetes, predicts cancer incidence and mortality. Future study on whether this relationship results from insulin resistance, the mechanisms underlying metabolic syndrome, or glucose variation may be a marker for metabolic syndrome is warranted.

In addition to its strengths, such as the relatively large number of patients with diabetes, a sufficiently long follow-up period, standardized procedure for data collection, and available information on a large number of potential confounding factors, this study is the first to use time-dependent annual glucose variations for exploring the relationship between glucose variation and cancer incidence and mortality. In addition, it is the first study that examines the relationship between cancer incidence and mortality and glucose variations among Chinese patients.

Our study is limited by potential residual and unrecognized confounding variables because our study is observational. In addition, measurement errors could be possible because of the large amount of data collected during clinical practice.

In summary, the annual FPG-CV may be included in the evaluation of glucose control in type 2 diabetic patients as a predictive factor for cancer incidence and mortality. Factors that may reduce FPG variations should be explored over time. Clinical trials with large sample sizes that involve interventions for maintaining a stable glycemia should provide conclusive evidence that glucose stability actually reduces cancer incidence and mortality. 


\section{Declaration of interest}

The authors declare that there is no conflict of interest that could be perceived as prejudicing the impartiality of the research reported.

\section{Funding}

This study was primarily supported by the National Science Council of Taiwan (NSC95-2314-B-039-009 and NSC972314-B-039-019), the China Medical University (CMU97176), the Taiwan Department of Health Clinical Trial and Research Center for Excellence (DOH101-TD-B-111-004), and Taiwan Department of Health, China Medical University Hospital Cancer Research Center of Excellence (DOH101TD-C-111-005).

\section{References}

Alberti KG, Zimmet P \& Shaw J 2006 Metabolic syndrome a new world-wide definition. A Consensus Statement from the International Diabetes Federation. Diabetic Medicine 23 469-480. (doi:10.1111/j.1464-5491.2006. 01858.x)

Bohlke K, Cramer DW, Trichopoulos D \& Mantzoros CS 1998 Insulin-like growth factor-1 in relation to premenopausal ductal carcinoma in situ of the breast. Epidemiology 9 570-573. (doi:10.1097/00001648199809000-00018)

Chan JM, Stampfer MJ, Giovannucci E, Gann PH, Ma J, Wilkinson P, Hennekens CH \& Pollak M 1998 Plasma insulin-like growth factor-1 and prostate cancer risk: a prospective study. Science 279 563-566. (doi:10.1126/ science.279.5350.563)

Cohen P, Clemmons DR \& Rosenfeld RG 2000 Does the GH-IGF axis play a role in cancer pathogenesis? Growth Hormone \& IGF Research 10 297-305. (doi:10.1054/ ghir.2000.0171)

Cust AE, Kaaks R, Friedenreich C, Bonnet F, Laville M, Tjønneland A, Olsen A, Overvad K, Jakobsen MU, Chajés V et al. 2007 Metabolic syndrome, plasma lipid, lipoprotein and glucose levels, and endometrial cancer risk in the European Prospective Investigation into Cancer and Nutrition (EPIC). Endocrine-Related Cancer 14 755-767. (doi:10.1677/ERC-07-0132)

El-Serag HB, Tran T \& Everhart JE 2004 Diabetes increases the risk of chronic liver disease and hepatocellular carcinoma. Gastroenterology 126 460-468. (doi:10.1053/ j.gastro.2003.10.065)

Flood A, Strayer L, Schairer C \& Schatzkin A 2010 Diabetes and risk of incident colorectal cancer in a prospective cohort of women. Cancer Causes \& Control 21 1277-1284. (doi:10.1007/s10552-010-9555-0)

Fujino Y, Mizoue T, Tokui N \& Yoshimura T 2001 Prospective study of diabetes mellitus and liver cancer in Japan. Diabetes/Metabolism Research and Reviews 17 374-379. (doi:10.1002/dmrr.214)
Greten FR, Eckmann L, Greten TF, Park JM, Li ZW, Egan LJ, Kagnoff MF \& Karin M 2004 IKK $\beta$ links inflammation and tumorigenesis in a mouse model of colitis-associated cancer. Cell 118 285-296. (doi:10.1016/j.cell.2004.07.013)

Grimberg A \& Cohen P 2000 Role of insulin-like growth factors and their binding proteins in growth control and carcinogenesis. Journal of Cellular Physiology 183 1-9. (doi:10.1002/(SICI)1097-4652(200004)183:1<1:: AID-JCP1 > 3.0.CO;2-J)

Gullo L, Pezzilli R \& Morselli-Labate AM 1994 Diabetes and the risk of pancreatic cancer. New England Journal of Medicine 331 81-84. (doi:10.1056/ NEJM199407143310203)

He J, Stram DO, Kolonel LN, Henderson BE, Le Marchand L \& Haiman CA 2010 The association of diabetes with colorectal cancer risk: the Multiethnic Cohort. British Journal of Cancer 103 120-126. (doi:10.1038/sj.bjc.6605721)

Hemminki K, Li X, Sundquist J \& Sundquist K 2010 Risk of cancer following hospitalization for type 2 diabetes. Oncologist 15 548-555. (doi:10.1634/theoncologist. 2009-0300)

Hu MC, Lee DF, Xia W, Golfman LS, Ou-Yang F, Yang JY, Zou Y, Bao S, Hanada N, Saso H et al. 2004 ІкB kinase promotes tumorigenesis through inhibition of forkhead FOXO3a. Cell 117 225-237. (doi:10.1016/S00928674(04)00302-2)

Kaaks R \& Lukanova A 2001 Energy balance and cancer: the role of insulin and insulin-like growth factor-I. Proceedings of the Nutrition Society 60 91-106. (doi:10.1079/PNS200070)

Kaaks R, Lukanova A \& Kurzer MS 2002 Obesity, endogenous hormones, and endometrial cancer risk: a synthetic review. Cancer Epidemiology, Biomarkers \& Prevention 11 1531-1543.

Larsson SC, Giovannucci E \& Wolk A 2005 Diabetes and colorectal cancer incidence in the cohort of Swedish men. Diabetes Care 28 1805-1807. (doi:10.2337/diacare. 28.7.1805)

Lee DF, Kuo HP, Chen CT, Hsu JM, Chou CK, Wei Y, Sun HL, Li LY, Ping B, Huang WC et al. 2004 IKK $\beta$ suppression of TSC1 links inflammation and tumor angiogenesis via the mTOR pathway. Cell $130440-455$. (doi:10.1016/j.cell.2007.05.058)

Lee DF, Kuo HP, Chen CT, Wei Y, Chou CK, Hung JY, Yen CJ \& Hung MC 2008 IKK $\beta$ suppression of TSC1 function links the mTOR pathway with insulin resistance. International Journal of Molecular Medicine 22 633-638. (doi:10.3892/ijmm_00000065)

Levine W, Dyer AR, Shekelle RB, Schoenberger JA \& Stamler J 1990 Post-load plasma glucose and cancer mortality in middle-aged men and women, 12-year follow-up findings of the Chicago Heart Association Detection Project in Industry. American Journal of Epidemiology 131 254-262.

Li Q, Kuriyama S, Kakizaki M, Yan H, Sone T, Nagai M, Sugawara Y, Ohmori-Matsuda K, Hozawa A, Nishino Y 
et al. 2010 History of diabetes mellitus and the risk of prostate cancer: the Ohsaki Cohort Study. Cancer Causes \& Control 21 1025-1032. (doi:10.1007/s10552-010-9530-9)

Limburg PJ, Vierkant RA, Fredericksen ZS, Leibson CL, Rizza RA, Gupta AK, Ahlquist DA, Melton LJ III, Sellers TA \& Cerhan JR 2006 Clinically confirmed type 2 diabetes mellitus and colorectal cancer risk: a populationbased, retrospective cohort study. American Journal of Gastroenterology 101 1872-1879. (doi:10.1111/j.15720241.2006.00725.x)

Mason PR 1999 Calcium channel blockers, apoptosis and cancer: is there a biologic relationship? Journal of the American College of Cardiology 34 1857-1866. (doi:10.1016/S0735-1097(99)00447-7)

Michels KB, Solomon CG, Hu FB, Rosner BA, Hankinson SE, Colditz GA, Manson JE \& Nurses' Health Study 2003 Type 2 diabetes and subsequent incidence of breast cancer in the Nurses' Health Study. Diabetes Care 26 1752-1758. (doi:10.2337/diacare.26.6.1752)

Mink PJ, Shahar E, Rosamond WD, Alberg AJ \& Folsom AR 2002 Serum insulin and glucose levels and breast cancer incidence: the atherosclerosis risk in communities study. American Journal of Epidemiology 156 349-352. (doi:10.1093/aje/kwf050)

Muggeo M, Verlato G, Bonora E, Ciani F, Moghetti P, Eastman R, Crepaldi G \& de Marco R 1995 Long-term instability of fasting plasma glucose predicts mortality in elderly NIDDM patients: the Verona Diabetes Study. Diabetologia 38 672-679. (doi:10.1007/BF00401838)

Muggeo M, Zoppini G, Bonora E, Brun E, Bonadonna RC, Moghetti P \& Verlato G 2000 Fasting plasma glucose variability predicts 10 -year survival of type 2 diabetic patients: the Verona Diabetes Study. Diabetes Care $\mathbf{2 3}$ 45-50. (doi:10.2337/diacare.23.1.45)

Nagamani M \& Stuart CA 1998 Specific binding and growthpromoting activity of insulin in endometrial cancer cells in culture. American Journal of Obstetrics and Gynaecology 179 6-12. (doi:10.1016/S0002-9378(98)70244-3)
Overvad K 2002 Body weight, physical activity and cancer. IARC Scientific Publications 156 235-236.

Rodriguez C, Patel AV, Mondul AM, Jacobs EJ, Thun MJ \& Calle EE 2005 Diabetes and risk of prostate cancer in a prospective cohort of US men. American Journal of Epidemiology 161 147-152. (doi:10.1093/ aje/kwh334)

Russo A, Autelitano M \& Bisanti L 2008 Metabolic syndrome and cancer risk. European Journal of Cancer 44 293-297. (doi:10.1016/j.ejca.2007.11.005)

Soler M, Chatenoud L, Negri E, Parazzini F, Franceschi S \& La Vecchia C 1999 Hypertension and hormonerelated neoplasms in women. Hypertension 34 320-325. (doi:10.1161/01.HYP.34.2.320)

Wang F, Herrington M, Larsson J \& Permert J 2003 The relationship between diabetes and pancreatic cancer. Molecular Cancer 2 4. (doi:10.1186/1476-4598-2-4)

Waters KM, Henderson BE, Stram DO, Wan P, Kolonel LN \& Haiman CA 2009 Association of diabetes with prostate cancer risk in the multiethnic cohort. American Journal of Epidemiology 169 937-945. (doi:10.1093/ aje/kwp003)

World Cancer Research Fund/American Institute for Cancer Research (WCRF/AICR) 1997 Food, Nutrition and the Prevention of Cancer: A Global Perspective. Washington, DC, WCRF/AICR.

Yun J, Rago C, Cheong I, Pagliarini R, Angenendt P, Rajagopalan H, Schmidt K, Willson JK, Markowitz S, Zhou S et al. 2009 Glucose deprivation contributes to the development of KRAS pathway mutations in tumor cells. Science 325 1555-1559. (doi:10.1126/ science.1174229)

Received in final form 24 April 2012

Accepted 26 April 2012

Made available online as an Accepted Preprint 27 April 2012 